\title{
Quantum tunneling splittings from path-integral molecular dynamics
}

\author{
Edit Mátyus, ${ }^{1}$ David J. Wales, ${ }^{1}$ and Stuart C. Althorpe ${ }^{1}$ \\ ${ }^{1}$ Department of Chemistry, University of Cambridge, \\ Lensfield Road, Cambridge, CB2 1EW, United Kingdom
}

(Dated: 29 February 2016)

\begin{abstract}
We illustrate how path-integral molecular dynamics (PIMD) can be used to calculate ground-state tunnelling splittings in molecules or clusters. The method obtains the splittings from ratios of density matrix elements between the degenerate wells connected by the tunnelling. We propose a simple thermodynamic integration scheme for evaluating these elements. Numerical tests on fullydimensional malonaldehyde yield tunnelling splittings in good overall agreement with the results of diffusion Monte Carlo calculations.
\end{abstract}




\section{INTRODUCTION}

The calculation of tunneling splittings allows non-equilibrium regions of a potential energy surface to be compared with spectroscopic data [1-24]. This comparison is of particular interest in water clusters, for which it is now possible to measure high resolution spectra [25-33] of rearrangement tunnelling in clusters with three-dimensional hydrogen-bonded structures, thus probing the intermolecular forces in water. Such systems are too large for direct solution of the Schrödinger equation (which can treat only the water dimer [10-14]), but can be addressed by two types of method in common use: diffusion Monte Carlo (DMC) [5-9, 34-36] (and related projection-operator techniques $[15,16,37,38]$ ), which is in principle exact, and instanton theory $[17-24,39]$, which is a semiclassical approximation.

In DMC, one exploits the resemblance of the imaginary-time Schrödinger equation to a diffusion equation, and uses Monte Carlo sampling to relax an initial distribution of walkers into the ground state; the calculation scales approximately linearly with system size. To calculate tunneling splittings, one needs to repeat this process for the first excited state, which is possible if one can locate the nodal dividing surface, or a good approximation to it (as is the case in malonaldehyde). However, in more complex 'floppy' systems, such as water clusters, the nodal dividing surfaces can be difficult to locate, accurately.

The instanton approach [17-24, 39-43] is also an imaginary-time based method. The tunnelling splitting is obtained from the 'instanton' - the classical path in imaginary time connecting the degenerate wells. The instanton is easy to find when the method is implemented in Cartesian coordinates, where it reduces to a linear segment of a (fictitious) 'ring-polymer' (such as is used in path-integral quantum simulations); minimizing the action is then equivalent to minimizing the (fictitious) energy of the linear polymer [22, 39]. This 'ring-polymer instanton' (RPI) method has been applied to a variety of water clusters, where it has correctly predicted the order-of-magnitude and pattern of the splittings $[23,24]$. Instanton methods have limited accuracy, because they approximate the density matrix elements by harmonic fluctuations around the minimum-action paths.

However, it is possible, at least in principle, to calculate the required matrix elements exactly, thus obtaining a method which combines the advantages of DMC (an exact quantum, linear scaling method) with RPI (which does not require the nodal surface). Methods capable of evaluating the matrix elements using path-integral Monte Carlo (PIMC) or path- 
integral molecular dynamics (PIMD) [44-46] were developed some time ago, in applications to one-dimensional model systems (PIMC) [47], crystal ${ }^{3} \mathrm{He}$ (PIMC) [48, 49], and to electron tunneling in proteins (PIMC) [50] and in $\mathrm{Fe}^{2+} / \mathrm{Fe}^{3+}$ (a combination of PIMC and PIMD) [51]. Here, we investigate whether it is practical to use the PIMD version of this approach to compute (ro)vibrational tunnelling splittings in molecules.

The paper is structured as follows. In Section II, we give the basic equations relating the tunnelling splitting to the density-matrix elements (introduced by previous workers). In Section III, we describe how to compute the matrix element ratios by PIMD, putting forward a simple thermodynamic integration scheme in Section IV. In Section V we report numerical applications, first to some simple 1-D examples, and then to the fully dimensional malonaldehyde molecule. Section VI concludes the article.

\section{RELATION OF THE TUNNELLING SPLITTING TO THE DENSITY MA- TRIX FOR A DOUBLE-WELL SYSTEM}

Here, we follow mostly the derivation outlined in Refs. [48, 49], pointing out such modifications as are necessary to apply it to tunnelling in a molecular system. The Hamiltonian of an $N$-particle system in $f$ spatial dimensions is

$$
\hat{H}=\sum_{i=1}^{f N} \frac{1}{2 m_{i}} \hat{p}_{i}^{2}+V(\boldsymbol{r})
$$

and the corresponding thermal density operator is written as

$$
\hat{\rho}=\mathrm{e}^{-\beta \hat{H}}
$$

where $1 / \beta=k_{\mathrm{B}} T$ is the temperature $T$ multiplied by the Boltzmann constant $k_{\mathrm{B}}$.

First, we consider the coordinate representation of the quantum thermal density matrix in terms of (bound-state) eigenvalues, $E_{n}$, and eigenfunctions, $\psi_{n}$, of the molecular Hamiltonian:

$$
\rho\left(\boldsymbol{r}, \boldsymbol{r}^{\prime} ; \beta\right)=\sum_{n} \psi_{n}^{*}(\boldsymbol{r}) \psi_{n}\left(\boldsymbol{r}^{\prime}\right) \mathrm{e}^{-\beta E_{n}}
$$


We assume that the double-well system has a single symmetry operator, $\hat{P}$, which acts on the Cartesian coordinates as $\boldsymbol{r}^{\prime}=\hat{P} \boldsymbol{r}=-\boldsymbol{r}$, and that the two lowest-energy eigenfunctions are symmetric and antisymmetric with respect to $\hat{P}: \psi_{0}\left(\boldsymbol{r}^{\prime}=-\boldsymbol{r}\right)=\psi_{0}(\boldsymbol{r})$ and $\psi_{1}\left(\boldsymbol{r}^{\prime}=\right.$ $-\boldsymbol{r})=-\psi_{1}(\boldsymbol{r})$, respectively. Then, the density matrix elements connecting symmetryrelated end points also carry this phase information induced by $\hat{P}$ :

$$
\begin{aligned}
\rho(\boldsymbol{r}, \boldsymbol{r} ; \beta) & =\left|\psi_{0}(\boldsymbol{r})\right|^{2} \mathrm{e}^{-\beta E_{0}}+\left|\psi_{1}(\boldsymbol{r})\right|^{2} \mathrm{e}^{-\beta E_{1}}+\ldots \\
\rho(\boldsymbol{r}, \hat{P} \boldsymbol{r} ; \beta) & =\psi_{0}^{*}(\boldsymbol{r}) \psi_{0}(\hat{P} \boldsymbol{r}) \mathrm{e}^{-\beta E_{0}}+\psi_{1}^{*}(\boldsymbol{r}) \psi_{1}(\hat{P} \boldsymbol{r}) \mathrm{e}^{-\beta E_{1}}+\ldots \\
& =\left|\psi_{0}(\boldsymbol{r})\right|^{2} \mathrm{e}^{-\beta E_{0}}-\left|\psi_{1}(\boldsymbol{r})\right|^{2} \mathrm{e}^{-\beta E_{1}}+\ldots
\end{aligned}
$$

For low temperatures we may neglect the contribution of the higher-energy $(n \geq 2)$ states. For sufficiently low temperature ("large $\beta$ ", defined by $\beta\left(E_{2}-E_{0}\right) \gg 1$ ) we can write:

$$
\begin{aligned}
\frac{\rho(\boldsymbol{r}, \hat{P} \boldsymbol{r} ; \beta)}{\rho(\boldsymbol{r}, \boldsymbol{r} ; \beta)} & \approx \frac{\left|\psi_{0}(\boldsymbol{r})\right|^{2} \mathrm{e}^{-\beta E_{0}}-\left|\psi_{1}(\boldsymbol{r})\right|^{2} \mathrm{e}^{-\beta E_{1}}}{\left|\psi_{0}(\boldsymbol{r})\right|^{2} \mathrm{e}^{-\beta E_{0}}+\left|\psi_{1}(\boldsymbol{r})\right|^{2} \mathrm{e}^{-\beta E_{1}}} \\
& =\frac{1-|\alpha(\boldsymbol{r})|^{2} \mathrm{e}^{-\beta \Delta E}}{1+|\alpha(\boldsymbol{r})|^{2} \mathrm{e}^{-\beta \Delta E}} \\
& =\frac{1-\mathrm{e}^{-\Delta E[\beta-\bar{\beta}(\boldsymbol{r})]}}{1+\mathrm{e}^{-\Delta E[\beta-\bar{\beta}(\boldsymbol{r})]}} \\
& =\tanh \left(\frac{1}{2} \Delta E[\beta-\bar{\beta}(\boldsymbol{r})]\right)
\end{aligned}
$$

where we introduced the tunneling (energy) splitting

$$
\Delta E=E_{1}-E_{0}
$$

and

$$
\bar{\beta}(\boldsymbol{r})=\frac{2}{\Delta E} \ln |\alpha(\boldsymbol{r})| \quad \text { with } \quad \alpha(\boldsymbol{r})=\frac{\psi_{1}(\boldsymbol{r})}{\psi_{0}(\boldsymbol{r})} .
$$

$\bar{\beta}(\boldsymbol{r})$ is inversely proportional to the energy splitting and also carries information about the relative amplitude of the eigenfunctions at $\boldsymbol{r}$; it can be interpreted as the "tunneling time", i.e. the amount of (imaginary) time necessary for the system to tunnel between the wells $[48,49]$. 
In principle, $\boldsymbol{r}$ is an arbitrary geometry at which the lowest two eigenfunctions have sufficient amplitude to carry the phase change induced by the symmetry operator. In practice, a simple and convenient choice of $\boldsymbol{r}$ is a structure near the equilibrium geometry of the PES. In the special case that $\tanh \left(\frac{1}{2} \Delta E(\beta-\bar{\beta}(\boldsymbol{r}))\right) \approx \frac{1}{2} \Delta E(\beta-\bar{\beta}(\boldsymbol{r}))$, the splitting $\Delta E$ can be directly calculated from the ratio $\rho(\boldsymbol{r}, \hat{P} \boldsymbol{r} ; \beta) / \rho(\boldsymbol{r}, \boldsymbol{r} ; \beta)$ evaluated at two different $\beta$ values. However, it is usually necessary to fit a hyperbolic tangent function to $\rho(\boldsymbol{r}, \hat{P} \boldsymbol{r} ; \beta) / \rho(\boldsymbol{r}, \boldsymbol{r} ; \beta)$ as a function of $\beta$ (which is what is done in the numerical tests of Sec. IV).

Clearly the use of Eq. (6) has much in common with the DMC method. However, as mentioned in the Introduction, Eq. (6) has the major advantage that, unlike DMC, it does not require knowledge of the entire nodal hypersurface of the upper state. Instead, it requires one to know the effect of the symmetry operation $\hat{P}$ on a single geometry $\boldsymbol{r}$ near to the minimum of one of the wells. A disadvantage (with respect to DMC) is that Eq. (6) yields only the splitting $\Delta E$, whereas DMC yields also the zero-point energy.

\section{CALCULATING THE DENSITY MATRIX ELEMENTS BY PIMD}

The tunneling splitting $\Delta E$ can be obtained from Eq. (6) if the low-temperature behaviour of the ratio of the density matrix elements is known. These elements and their corresponding ratio can be calculated efficiently in the discretized path-integral formalism, which translates the quantum mechanical problem to a classical phase-space integral of a hypothetical linear polymer. In what follows we introduce the necessary expressions for the calculation of these density-matrix elements with path integrals and outline the main elements of our algorithm and numerical implementation. We develop the formalism neglecting the spin of the particles, because the density matrix is used only as a mathematical and computational tool, which gives access to the energy splitting through Eq. (6). The quantum statistical weights of the physical particles can be assigned to the energy levels at the end of the calculation. Then, a density matrix element is written in the position representation and the resolution of the identity is inserted $(M-1)$ times

$$
\rho(\boldsymbol{a}, \boldsymbol{b} ; \beta)=\int \ldots \int \mathrm{d} \boldsymbol{r}^{(1)} \ldots \mathrm{d} \boldsymbol{r}^{(M-1)} \rho\left(\boldsymbol{a}, \boldsymbol{r}^{(1)} ; \beta_{M}\right) \ldots \rho\left(\boldsymbol{r}^{(M-1)}, \boldsymbol{b} ; \beta_{M}\right)
$$


with $\beta_{M}=\beta / M$. Using Trotter's theorem we can approximate the high-temperature (small $\left.\beta_{M}\right)$ density matrix as

$$
\begin{aligned}
\rho\left(\boldsymbol{r}, \boldsymbol{r}^{\prime} ; \beta_{M}\right) \approx & \left(\frac{1}{2 \pi \hbar^{2} \beta_{M}}\right)^{f N / 2}\left(\prod_{i=1}^{f N} m_{i}\right)^{1 / 2} \\
& \exp \left[-\beta_{M}\left(\sum_{i=1}^{f N} \frac{1}{2} m_{i} \omega_{M}^{2}\left(r_{i}-r_{i}^{\prime}\right)^{2}+\frac{1}{2}\left(V(\boldsymbol{r})+V\left(\boldsymbol{r}^{\prime}\right)\right)\right)\right]
\end{aligned}
$$

with $\omega_{M}=1 /\left(\hbar \beta_{M}\right)$. By inserting Eq. (10) into Eq. (9), $\rho(\boldsymbol{a}, \boldsymbol{b} ; \beta)$ can be approximated as

$$
\begin{aligned}
& \rho(\boldsymbol{a}, \boldsymbol{b} ; \beta) \approx \rho_{M}(\boldsymbol{a}, \boldsymbol{b} ; \beta) \\
& =\left(\frac{1}{2 \pi \hbar^{2} \beta_{M}}\right)^{f N M / 2}\left(\prod_{i=1}^{f N} m_{i}\right)^{M / 2} \exp \left[-\beta_{M}\left(V\left(\boldsymbol{r}^{(0)}\right)+V\left(\boldsymbol{r}^{(M)}\right)\right) / 2\right] \\
& \int \ldots \int \mathrm{d} \boldsymbol{r}^{(1)} \ldots \mathrm{d} \boldsymbol{r}^{(M-1)} \exp \left[-\beta_{M}\left\{\sum_{j=1}^{M} \sum_{i=1}^{f N} \frac{1}{2} m_{i} \omega_{M}^{2}\left(r_{i}^{(j)}-r_{i}^{(j-1)}\right)^{2}+\sum_{j=1}^{M-1} V\left(\boldsymbol{r}^{(j)}\right)\right\}\right] \\
& \text { with } \quad \boldsymbol{r}^{(0)}=\boldsymbol{a} \quad \text { and } \quad \boldsymbol{r}^{(M)}=\boldsymbol{b}
\end{aligned}
$$

where in the large $M$ limit the exact value is obtained, $\lim _{M \rightarrow \infty} \rho_{M}(\boldsymbol{a}, \boldsymbol{b} ; \beta)=\rho(\boldsymbol{a}, \boldsymbol{b} ; \beta)$. Furthermore, we can insert the identity

$$
\left(\frac{\beta_{M}}{2 \pi \mu_{i}^{(j)}}\right)^{1 / 2} \int_{-\infty}^{\infty} \mathrm{d} p_{i}^{(j)} \exp \left[-\beta_{M} \frac{\left(p_{i}^{(j)}\right)^{2}}{2 \mu_{i}^{(j)}}\right]=1, \quad \mu_{i}^{(j)}>0
$$

into Eq. (11) an arbitrary number of times. For later convenience, we introduce $f N(M-1)$ new variables in the expression using Eq. (12) for $i=1,2, \ldots, f N$ and $j=1,2, \ldots, M-1$, and the density matrix element is written as

$$
\begin{aligned}
& \rho_{M}(\boldsymbol{a}, \boldsymbol{b} ; \beta)=\frac{1}{h^{f N M}}\left(\prod_{i=1}^{f N} \frac{2 \pi m_{i}^{1 / 2}}{\beta_{M}}\right)\left(\prod_{i=1}^{f N} \prod_{j=1}^{M-1} \frac{m_{i}}{\mu_{i}^{(j)}}\right)^{1 / 2} \\
& \int \ldots \int \mathrm{d} \boldsymbol{p}^{(1)} \ldots \mathrm{d} \boldsymbol{p}^{(M-1)} \int \ldots \int \mathrm{d} \boldsymbol{r}^{(1)} \ldots \mathrm{d} \boldsymbol{r}^{(M-1)} \exp \left[-\beta_{M} H_{\boldsymbol{a}, \boldsymbol{b}}^{[M-1]}\left(\boldsymbol{p}, \boldsymbol{r} ; \beta_{M}\right)\right],
\end{aligned}
$$

which, similarly to Eq. (11), is equal to $\rho(\boldsymbol{a}, \boldsymbol{b}, \beta)$ in the $M \rightarrow \infty$ limit. Equation (13) is a phase-space integral for a hypothetical linear polymer described by the Hamiltonian 
$H_{\boldsymbol{a}, \boldsymbol{b}}^{[M-1]}\left(\boldsymbol{p}, \boldsymbol{r} ; \beta_{M}\right)$ corresponding to fixed-end beads, at $\boldsymbol{a}$ and at $\boldsymbol{b}$,

$$
H_{\boldsymbol{a}, \boldsymbol{b}}^{[M-1]}\left(\boldsymbol{p}, \boldsymbol{r} ; \beta_{M}\right)=\mathcal{H}_{\boldsymbol{a}, \boldsymbol{b}}^{[M-1]}\left(\boldsymbol{p}, \boldsymbol{r} ; \beta_{M}\right)+\mathcal{V}^{[M-1]}(\boldsymbol{r})+(V(\boldsymbol{a})+V(\boldsymbol{b})) / 2 .
$$

$\mathcal{H}_{\boldsymbol{a}, \boldsymbol{b}}^{[M-1]}\left(\boldsymbol{p}, \boldsymbol{r} ; \beta_{M}\right)$ is the corresponding free linear-polymer Hamiltonian

$$
\begin{aligned}
& \mathcal{H}_{\boldsymbol{a}, \boldsymbol{b}}^{[M-1]}\left(\boldsymbol{p}, \boldsymbol{r} ; \beta_{M}\right)=\left.\sum_{i=1}^{f N}\left[\sum_{j=1}^{M-1} \frac{1}{2 \mu_{i}^{(j)}}\left(p_{i}^{(j)}\right)^{2}+\sum_{j=1}^{M} \frac{1}{2} m_{i} \omega_{M}^{2}\left(r_{i}^{(j)}-r_{i}^{(j-1)}\right)^{2}\right]\right|_{\substack{\boldsymbol{r}^{(0)}=\boldsymbol{a} \\
\boldsymbol{r}^{(M)}=\boldsymbol{b}}} \\
&= \sum_{i=1}^{f N}\left[\sum_{j=1}^{M-1} \frac{1}{2 \mu_{i}^{(j)}}\left(p_{i}^{(j)}\right)^{2}+\sum_{j=2}^{M-1} \frac{1}{2} m_{i} \omega_{M}^{2}\left(r_{i}^{(j)}-r_{i}^{(j-1)}\right)^{2}\right. \\
&+\left.\frac{1}{2} m_{i} \omega_{M}^{2}\left(r_{i}^{(1)}-a_{i}\right)^{2}+\frac{1}{2} m_{i} \omega_{M}^{2}\left(b_{i}-r_{i}^{(M-1)}\right)^{2}\right] \\
& \text { with } \quad \omega_{M}=1 /\left(\hbar \beta_{M}\right)
\end{aligned}
$$

which contains $(M-1)$ beads (copies of the system) connected by springs of a force constant $m_{i} \omega_{M}^{2}(i=1,2, \ldots, f N)$. The sum of the potential energy of all the active beads is

$$
\mathcal{V}^{[M-1]}(\boldsymbol{r})=\sum_{j=1}^{M-1} V\left(\boldsymbol{r}^{(j)}\right)
$$

The bead masses, $\mu_{i}^{(j)}$ (the Parrinello-Rahman "mass matrix" [46]), can be chosen arbitrarily and certain choices are motivated by physical or computational arguments. A simple choice is to have the same mass for all beads corresponding to the same degree of freedom and equal or proportional to the physical mass of the corresponding particle $\mu_{i}^{(1)}=\mu_{i}^{(2)}=\ldots=$ $\mu_{i}^{(M-1)}=\mu_{i}=m_{i} / \tilde{\mu}_{i}(i=1,2, \ldots, f N)$ (with same mass scaling coefficient, $\tilde{\mu}_{i}$, introduced for each degree of freedom). Another choice of bead masses is introduced in Sec. IVB, which gives details of the numerical tests. 


\section{THERMODYNAMIC INTEGRATION OF THE DENSITY-MATRIX ELE- MENT RATIO}

The ratio of two elements of the density matrix necessary to calculate the energy splitting, Eq. (6), using the discretized path-integral formalism, Eq. (13), is written as

$$
\begin{aligned}
& \frac{\rho(\boldsymbol{a}, \boldsymbol{b} ; \beta)}{\rho(\boldsymbol{a}, \boldsymbol{a} ; \beta)} \approx \frac{\rho_{M}(\boldsymbol{a}, \boldsymbol{b} ; \beta)}{\rho_{M}(\boldsymbol{a}, \boldsymbol{a} ; \beta)} \\
& =\frac{\int \ldots \int \mathrm{d} \boldsymbol{p}^{(1)} \ldots \mathrm{d} \boldsymbol{p}^{(M-1)} \int \ldots \int \mathrm{d} \boldsymbol{r}^{(1)} \ldots \mathrm{d} \boldsymbol{r}^{(M-1)} \exp \left[-\beta_{M} H_{\boldsymbol{a}, \boldsymbol{b}}^{[M-1]}\left(\boldsymbol{p}, \boldsymbol{r} ; \beta_{M}\right)\right]}{\int \ldots \int \mathrm{d} \boldsymbol{p}^{(1)} \ldots \mathrm{d} \boldsymbol{p}^{(M-1)} \int \ldots \int \mathrm{d} \boldsymbol{r}^{(1)} \ldots \mathrm{d} \boldsymbol{r}^{(M-1)} \exp \left[-\beta_{M} H_{\boldsymbol{a}, \boldsymbol{a}}^{[M-1]}\left(\boldsymbol{p}, \boldsymbol{r} ; \beta_{M}\right)\right]}
\end{aligned}
$$

(for large $M$ )

In principle, Eq. (17) could be evaluated by using an approach similar to the free energy perturbation method [52] by calculating the exponentiated difference of the Hamiltonians over a canonical ensemble corresponding to the parameters $(\boldsymbol{a}, \boldsymbol{a} ; \beta, M)$. However, this simple approach is impractical unless $\boldsymbol{b}$ is very close to $\boldsymbol{a}$, which is usually not true for structures related by discrete symmetry operations. Instead, we use thermodynamic integration to evaluate Eq. (17).

Since $\rho_{M}(\boldsymbol{a}, \boldsymbol{b} ; \beta) \geq 0$, we can define a free energy function

$$
\mathcal{A}\left(\xi ; \beta_{M}\right)=-\frac{1}{\beta_{M}} \ln \rho_{M}(\boldsymbol{a}, \boldsymbol{b}(\xi) ; \beta)
$$

Then, we can write

$$
\begin{aligned}
\frac{\rho_{M}(\boldsymbol{a}, \boldsymbol{b} ; \beta)}{\rho_{M}(\boldsymbol{a}, \boldsymbol{a} ; \beta)} & =\frac{\rho_{M}\left(\boldsymbol{a}, \boldsymbol{b}\left(\xi_{1}\right) ; \beta\right)}{\rho_{M}\left(\boldsymbol{a}, \boldsymbol{b}\left(\xi_{0}\right) ; \beta\right)} \\
& =\exp \left[-\beta_{M}\left(\mathcal{A}\left(\xi_{1} ; \beta_{M}\right)-\mathcal{A}\left(\xi_{0} ; \beta_{M}\right)\right)\right] \\
& =\exp \left[-\beta_{M} \Delta \mathcal{A}\left(\xi: \xi_{0} \rightarrow \xi_{1} ; \beta_{M}\right)\right]
\end{aligned}
$$

where $\xi \in\left[\xi_{0}, \xi_{1}\right]$ is a reaction coordinate, which connects $\boldsymbol{b}\left(\xi_{0}\right)=\boldsymbol{a}$ with $\boldsymbol{b}\left(\xi_{1}\right)=\boldsymbol{b}$ along a smooth path. In the simplest case $\xi$ can be a linear interpolating function between $\boldsymbol{a}$ and $\boldsymbol{b}$. A more general choice of $\xi$ might be the integrated path length [53].

In what follows, we develop the thermodynamic integral for the free energy difference 
$\Delta \mathcal{A}\left(\xi: \xi_{0} \rightarrow \xi_{1} ; \beta_{M}\right)=\mathcal{A}\left(\xi_{1} ; \beta_{M}\right)-\mathcal{A}\left(\xi_{0} ; \beta_{M}\right)$ and thereby for

$$
\begin{aligned}
-\frac{1}{\beta_{M}} \ln \frac{\rho_{M}(\boldsymbol{a}, \boldsymbol{b} ; \beta)}{\rho_{M}(\boldsymbol{a}, \boldsymbol{a} ; \beta)} & =-\frac{1}{\beta_{M}} \ln \frac{\rho_{M}\left(\boldsymbol{a}, \boldsymbol{b}\left(\xi_{1}\right) ; \beta\right)}{\rho_{M}\left(\boldsymbol{a}, \boldsymbol{b}\left(\xi_{0}\right) ; \beta\right)} \\
& =\Delta \mathcal{A}\left(\xi: \xi_{0} \rightarrow \xi_{1} ; \beta_{M}\right) .
\end{aligned}
$$

To make the presentation more compact we introduce the shorthand notation

$$
\begin{aligned}
\rho_{M}(\xi ; \beta) & =\rho_{M}(\boldsymbol{a}, \boldsymbol{b}(\xi) ; \beta) \\
& =\int \ldots \int \mathrm{d} \boldsymbol{p}^{(1)} \ldots \mathrm{d} \boldsymbol{p}^{(M-1)} \int \ldots \int \mathrm{d} \boldsymbol{r}^{(1)} \ldots \mathrm{d} \boldsymbol{r}^{(M-1)} \exp \left[-\beta_{M} H_{\boldsymbol{a}, \boldsymbol{b}(\xi)}^{[M-1]}\left(\boldsymbol{p}, \boldsymbol{r} ; \beta_{M}\right)\right] \\
& =\int \ldots \int \mathrm{d} \boldsymbol{p}^{(1)} \ldots \mathrm{d} \boldsymbol{p}^{(M-1)} \int \ldots \int \mathrm{d} \boldsymbol{r}^{(1)} \ldots \mathrm{d} \boldsymbol{r}^{(M-1)} \exp \left[-\beta_{M} H_{\xi}^{[M-1]}\right] .
\end{aligned}
$$

So, the free energy difference can be written as

$$
\begin{aligned}
\Delta \mathcal{A}\left(\xi: \xi_{0} \rightarrow \xi_{1} ; \beta_{M}\right) & =\int_{\xi_{0}}^{\xi_{1}} \mathrm{~d} \xi^{\prime} \frac{\partial \mathcal{A}}{\partial \xi}\left(\xi^{\prime}\right) \\
& =\int_{\xi_{0}}^{\xi_{1}} \mathrm{~d} \xi^{\prime}\left\langle\frac{\partial H_{\xi}^{[M-1]}}{\partial \xi}\right\rangle_{\left(\xi=\xi^{\prime}\right)} \\
& =\int_{\xi_{0}}^{\xi_{1}} \mathrm{~d} \xi^{\prime}\left\langle\left.\frac{\partial H_{\boldsymbol{a}, \boldsymbol{b}(\xi)}^{[M-1]}\left(\boldsymbol{p}, \boldsymbol{r} ; \beta_{M}\right)}{\partial \xi}\right|_{\xi=\xi^{\prime}}\right\rangle_{\left(\boldsymbol{a}, \boldsymbol{b}\left(\xi^{\prime}\right) ; \beta, M\right)}
\end{aligned}
$$

where in the last step we reintroduced the fully detailed notation according to Eq. (21). This result means that the change in the free energy, $\Delta \mathcal{A}$, corresponding to pulling one of the ends of the linear polymer from $\boldsymbol{b}\left(\xi_{0}\right)$ to $\boldsymbol{b}\left(\xi_{1}\right)$ can be evaluated by integrating from $\xi_{0}$ to $\xi_{1}$ the potential of mean force (the expectation value of $\partial H_{\boldsymbol{a}, \boldsymbol{b}(\xi)}^{[M-1]} / \partial \xi$ over the ensembles along the path). We can write out the derivative of the full linear-polymer Hamiltonian, Eq. (14), as follows

$$
\begin{aligned}
\left.\frac{\partial H_{\boldsymbol{a}, \boldsymbol{b}(\xi)}^{[M-1]}\left(\boldsymbol{p}, \boldsymbol{r} ; \beta_{M}\right)}{\partial \xi}\right|_{\xi=\xi^{\prime}} & =\left.\sum_{i=1}^{f N} \frac{\partial H_{\boldsymbol{a}, \boldsymbol{b}(\xi)}^{[M-1]}\left(\boldsymbol{p}, \boldsymbol{r} ; \beta_{M}\right)}{\partial r_{i}^{(M)}} \frac{\partial r_{i}^{(M)}}{\partial \xi}\right|_{\xi^{\prime}} \\
& =\left.\sum_{i=1}^{f N}\left[\left.\frac{1}{2} \frac{\partial V}{\partial r_{i}}\right|_{r_{i}^{(M)}\left(\xi^{\prime}\right)}+m_{i} \omega_{M}^{2}\left(r_{i}^{(M)}\left(\xi^{\prime}\right)-r_{i}^{(M-1)}\right)\right] \frac{\partial r_{i}^{(M)}}{\partial \xi}\right|_{\xi^{\prime}}
\end{aligned}
$$


where $\boldsymbol{r}^{(M)}\left(\xi^{\prime}\right)=\boldsymbol{b}\left(\xi^{\prime}\right)$ is a parameter of the Hamiltonian along the reaction path connecting $\boldsymbol{a}$ and $\boldsymbol{b}$. By inserting Eqs. (22) and (23) into Eq. (20) we obtain

$$
\begin{aligned}
& -\frac{1}{\beta_{M}} \ln \frac{\rho_{M}(\boldsymbol{a}, \boldsymbol{b} ; \beta)}{\rho_{M}(\boldsymbol{a}, \boldsymbol{a} ; \beta)} \\
& =\left.\left.\int_{\xi_{0}}^{\xi_{1}} \mathrm{~d} \xi^{\prime} \sum_{i=1}^{f N} \frac{1}{2} \frac{\partial V}{\partial r_{i}}\right|_{r_{i}^{(M)}\left(\xi^{\prime}\right)} \frac{\partial r_{i}^{(M)}}{\partial \xi}\right|_{\xi^{\prime}} \\
& +\int_{\xi_{0}}^{\xi_{1}} \mathrm{~d} \xi^{\prime} \sum_{i=1}^{f N} m_{i} \omega_{M}^{2}\left\langle\left.\left(r_{i}^{(M)}\left(\xi^{\prime}\right)-r_{i}^{(M-1)}\right) \frac{\partial r_{i}^{(M)}}{\partial \xi}\right|_{\xi^{\prime}}\right\rangle_{\left(\boldsymbol{a}, \boldsymbol{b}\left(\xi^{\prime}\right) ; \beta, M\right)}
\end{aligned}
$$

The first term in Eq. (24) simplifies to

$$
\left.\left.\left.\int_{\xi_{0}}^{\xi_{1}} \mathrm{~d} \xi^{\prime} \sum_{i=1}^{f N} \frac{1}{2} \frac{\partial V}{\partial r_{i}}\right|_{r_{i}^{(M)}\left(\xi^{\prime}\right)} \frac{\partial r_{i}^{(M)}}{\partial \xi}\right|_{\xi^{\prime}}=\left.\int_{\xi_{0}}^{\xi_{1}} \mathrm{~d} \xi^{\prime} \frac{1}{2} \frac{\partial V}{\partial \xi}\right|_{\xi^{\prime}}=\frac{1}{2}(V(\boldsymbol{b})-V(\boldsymbol{a}))\right)=0
$$

if the initial and the final structure are related by a symmetry operation, $\boldsymbol{b}=\hat{P} \boldsymbol{a}$, which leaves the potential energy invariant, $V(\boldsymbol{a})=V(\hat{P} \boldsymbol{a})$. The second term in Eq. (24) can be written as

$$
\begin{aligned}
& \int_{\xi_{0}}^{\xi_{1}} \mathrm{~d} \xi^{\prime} \sum_{i=1}^{f N} m_{i} \omega_{M}^{2}\left\langle\left.\left(r_{i}^{(M)}\left(\xi^{\prime}\right)-r_{i}^{(M-1)}\right) \frac{\partial r_{i}^{(M)}}{\partial \xi}\right|_{\xi^{\prime}}\right\rangle_{\left(\boldsymbol{a}, \boldsymbol{b}\left(\xi^{\prime}\right) ; \beta, M\right)} \\
& =\left.\sum_{i=1}^{f N} m_{i} \omega_{M}^{2} \int_{\xi_{0}}^{\xi_{1}} \mathrm{~d} \xi^{\prime} \frac{\partial r_{i}^{(M)}}{\partial \xi}\right|_{\xi^{\prime}} r_{i}^{(M)}\left(\xi^{\prime}\right)-\left.\sum_{i=1}^{f N} m_{i} \omega_{M}^{2} \int_{\xi_{0}}^{\xi_{1}} \mathrm{~d} \xi^{\prime} \frac{\partial r_{i}^{(M)}}{\partial \xi}\right|_{\xi^{\prime}}\left\langle r_{i}^{(M-1)}\right\rangle_{\left(\boldsymbol{a}, \boldsymbol{b}\left(\xi^{\prime}\right) ; \beta, M\right)} \\
& =K-\frac{1}{\hbar^{2} \beta_{M}^{2}} \int_{\xi_{0}}^{\xi_{1}} \mathrm{~d} \xi\left[\frac{\partial \tilde{\boldsymbol{r}}^{(M)}}{\partial \xi}\right]^{\mathrm{T}}\left\langle\tilde{\boldsymbol{r}}^{(M-1)}\right\rangle_{(\boldsymbol{a}, \boldsymbol{b}(\xi) ; \beta, M)}
\end{aligned}
$$

where in the first step we note that $r_{i}^{(M)}\left(\xi^{\prime}\right)$ and $\partial r_{i}^{(M)} / \partial \xi$ give only a constant contribution to the expectation value because the end-bead is fixed. To shorten the notation we introduced 


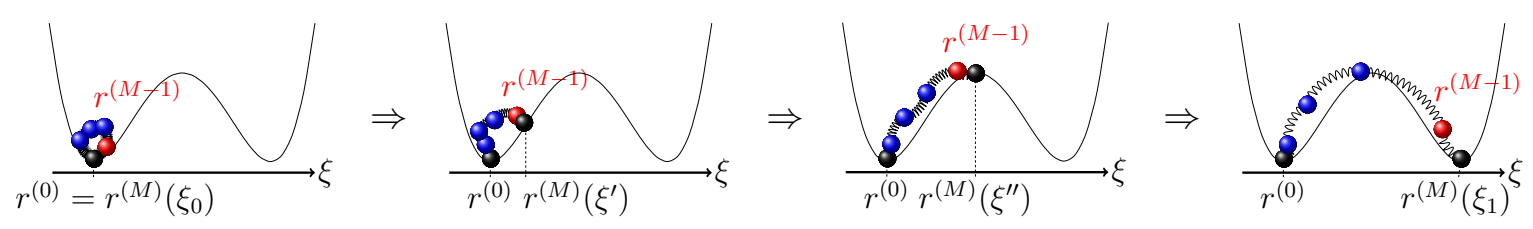

FIG. 1: The density matrix ratio, $\rho(\boldsymbol{a}, \boldsymbol{b} ; \beta) / \rho(\boldsymbol{a}, \boldsymbol{a} ; \beta)$ is determined from the coordinates of the last-but-one bead (red) upon pulling one end of the linear polymer from $\boldsymbol{a}$ to $\boldsymbol{b}$, Eq. (28). The end beads (black), $\boldsymbol{r}^{(0)}=a$ and $\boldsymbol{r}^{(M)}(\xi)$, are fixed in each simulation window (only four windows are shown).

mass-weighted coordinates, $\tilde{r}_{i}^{(j)}=\sqrt{m_{i}} r_{i}^{(j)}(i=1, \ldots, f N)$, and the constant $K$,

$$
\begin{aligned}
K & =\left.\frac{1}{\hbar^{2} \beta_{M}^{2}} \sum_{i=1}^{f N} \int_{\xi_{0}}^{\xi_{1}} \mathrm{~d} \xi^{\prime} \frac{\partial \tilde{r}_{i}^{(M)}}{\partial \xi}\right|_{\xi^{\prime}} \tilde{r}_{i}^{(M)}\left(\xi^{\prime}\right) \\
& =\frac{1}{2 \hbar^{2} \beta_{M}^{2}} \sum_{i=1}^{f N}\left(\tilde{b}_{i}^{2}-\tilde{a}_{i}^{2}\right)=\frac{1}{2 \hbar^{2} \beta_{M}^{2}} \sum_{i=1}^{f N}\left(\tilde{\boldsymbol{b}}^{\mathrm{T}} \tilde{\boldsymbol{b}}-\tilde{\boldsymbol{a}}^{\mathrm{T}} \tilde{\boldsymbol{a}}\right)=0,
\end{aligned}
$$

where $K=0$ holds for symmetry-related end points, since identical particles have the same mass and the scalar product is invariant to rotation-inversion operations $\left(\tilde{a}_{i}\right.$ and $\tilde{b}_{i}$ label mass-weighted coordinates, as introduced earlier, for the end points).

Using Eqs. (24)-(27) we can write the ratio of the density matrix elements, which connect symmetry-related structures, as

$$
\begin{array}{r}
\frac{\rho_{M}(\boldsymbol{a}, \boldsymbol{b} ; \beta)}{\rho_{M}(\boldsymbol{a}, \boldsymbol{a} ; \beta)}=\exp \left[\frac{1}{\hbar^{2} \beta_{M}} \int_{\xi_{0}}^{\xi_{1}} \mathrm{~d} \xi\left[\frac{\partial \tilde{\boldsymbol{r}}^{(M)}}{\partial \xi}\right]^{\mathrm{T}}\left\langle\tilde{\boldsymbol{r}}^{(M-1)}\right\rangle_{(\boldsymbol{a}, \boldsymbol{b}(\xi) ; \beta, M)}\right] \\
\left(\text { with } \boldsymbol{b}=\boldsymbol{b}\left(\xi_{1}\right)=\hat{P} \boldsymbol{a}\right) .
\end{array}
$$

This is our final working equation for the density matrix ratio. It is interesting to note that the ratio depends on the position expectation value of just the last-but-one bead as the last bead is pulled from structure $\boldsymbol{a}$ to the symmetry-related structure, $\boldsymbol{b}=\hat{P} \boldsymbol{a}$ (Figure 1). This may lead to inefficient sampling statistics, and suggests that future work should look for alternative integration pathways, which depend on the coordinates of all the beads. It is also interesting to note that the contribution of the potential energy (gradient) to the integral of the the potential of mean force, Eqs. (22) and (23), cancels for symmetry-related end points. 


\section{A. Path-integral molecular dynamics for the linear polymer}

Using Eq. (28), the ratio of the density matrix elements is obtained from the expectation value

$$
\begin{aligned}
& \left\langle r_{i}^{(M-1)}\right\rangle_{(\boldsymbol{a}, \boldsymbol{b}(\xi) ; \beta, M)}=\frac{1}{\rho_{M}(\boldsymbol{a}, \boldsymbol{b}(\xi), \beta)} \\
& \times \int \ldots \int \mathrm{d} \boldsymbol{p}^{(1)} \ldots \mathrm{d} \boldsymbol{p}^{(M-1)} \int \ldots \int \mathrm{d} \boldsymbol{r}^{(1)} \ldots \mathrm{d} \boldsymbol{r}^{(M-1)} r_{i}^{(M-1)} \exp \left[-\beta_{M} H_{\boldsymbol{a}, \boldsymbol{b}(\xi)}^{[M-1]}\left(\boldsymbol{p}, \boldsymbol{r} ; \beta_{M}\right)\right]
\end{aligned}
$$

with

$$
\begin{aligned}
& \rho_{M}(\boldsymbol{a}, \boldsymbol{b}(\xi), \beta) \\
& =\int \ldots \int \mathrm{d} \boldsymbol{p}^{(1)} \ldots \mathrm{d} \boldsymbol{p}^{(M-1)} \int \ldots \int \mathrm{d} \boldsymbol{r}^{(1)} \ldots \mathrm{d} \boldsymbol{r}^{(M-1)} \exp \left[-\beta_{M} H_{\boldsymbol{a}, \boldsymbol{b}(\xi)}^{[M-1]}\left(\boldsymbol{p}, \boldsymbol{r} ; \beta_{M}\right)\right] .
\end{aligned}
$$

This expectation value is evaluated using molecular dynamics sampling connected with, in our present implementation, an Andersen thermostat [54] (i.e., randomly selecting $\boldsymbol{p}$ from the Boltzmann distribution).

\section{B. Integrating the equations of motion}

The classical equations of motion corresponding to the linear-polymer Hamiltonian $H_{\boldsymbol{a}, \boldsymbol{b}(\xi)}^{[M-1]}$, Eq. (14), are integrated similarly to Eqs. (21)-(25) of ref. [55] but the ring-polymer normal modes are replaced with those of the linear polymer (Appendix A). Similarly to ref. [55], the total Hamiltonian is split into the exactly integrable parts of the free (linear) polymer and the potential energy contribution.

In order to make the sampling more efficient, instead of using the linear-polymer Hamiltonian $H_{\boldsymbol{a}, \boldsymbol{b}(\xi)}^{[M-1]}$, Eq. (14), in Cartesian coordinates, we use a related Hamiltonian, which is written in terms of the normal coordinates of the free linear polymer corresponding to the fixed end beads, $\boldsymbol{a}$ and $\boldsymbol{b}$,

$$
H_{\boldsymbol{a}, \boldsymbol{b}(\xi)}^{[M-1]^{\prime}}=\sum_{i=1}^{f N}\left[\sum_{j=1}^{M-1} \frac{1}{2}\left(\Pi_{i}^{(j)}\right)^{2}+\sum_{j=1}^{M-1} \frac{1}{2} m_{i} \omega_{M}^{2} \lambda^{(j)}\left(\eta_{i}^{(j)}\right)^{2}\right]
$$


where $\lambda^{(j)}$ and $\eta_{i}^{(j)}$ are defined in Eqs. (A.10), (A.18) and $\Pi_{i}^{(j)}$ are the corresponding normalcoordinate momenta. This Hamiltonian differs from $H_{\boldsymbol{a}, \boldsymbol{b}(\xi)}^{[M-1]}$ by a constant term, which is dependent on the end-bead coordinates (if the coordinates of the end beads are zero, this term is also zero), but it leads to equations of motion equivalent to those of the Cartesian Hamiltonian (the coordinates are related by a linear transformation and the Jacobian is unity). Next, we can introduce scaled normal coordinate momenta, $\tilde{\Pi}_{i}^{(j)}$

$$
\tilde{H}_{\boldsymbol{a}, \boldsymbol{b}(\xi)}^{[M-1]^{\prime}}=\sum_{i=1}^{f N}\left[\sum_{j=1}^{M-1} \frac{1}{2 M_{i}^{(j)}}\left(\tilde{\Pi}_{i}^{(j)}\right)^{2}+\sum_{j=1}^{M-1} \frac{1}{2} m_{i} \omega_{M}^{2} \lambda^{(j)}\left(\eta_{i}^{(j)}\right)^{2}\right]
$$

with some $M_{i}^{(j)}>0$ "bead-dependent" masses. We choose

$$
M_{i}^{(j)}=m_{i} \omega_{M}^{2} \lambda^{(j)}=4 m_{i} \omega_{M}^{2} \sin ^{2}\left(\frac{j \pi}{2 M}\right)
$$

and thus the scaled normal coordinates vibrate with the same angular frequency

$$
\tilde{\Omega}=\left(\tilde{\Omega}_{i}^{(j)}\right)^{2}=\frac{1}{M_{i}^{(j)}} m_{i} \omega_{M}^{2} \lambda^{(j)}=1
$$

We are free to use the equations of motion corresponding to the scaled Hamiltonian of Eq. (32) to sample the configuration space part of the integral, because the momentum space part of the integral factorises out. The effect of this scaling is equivalent to using a multiple time-step integration scheme for the equations of motions corresponding to Eqs. (14) or (31). Therefore, we can evaluate $\left\langle r_{i}^{(M-1)}\right\rangle_{\boldsymbol{a}, \boldsymbol{b}(\xi) ; \beta, M}$ in Eq. (29) using the equations of motion of $\tilde{H}_{\boldsymbol{a}, \boldsymbol{b}(\xi)}^{[M-1)^{\prime}}$, Eq. (32), to sample the integral more efficiently. At every time step the coordinates of the last-but-one bead $r_{i}^{(M-1)}$ are evaluated from the normal coordinates, $\eta_{i}^{(j)}$, using Eq. (A.19). The introduction of scaled bead masses in the normal mode representation does not hinder us from working with analytic expressions for carrying out the transformation between the normal and the Cartesian coordinates, Eqs. (A.18)-(A.19), and it can be evaluated efficiently for a large bead number using a fast sine-transform routine. 

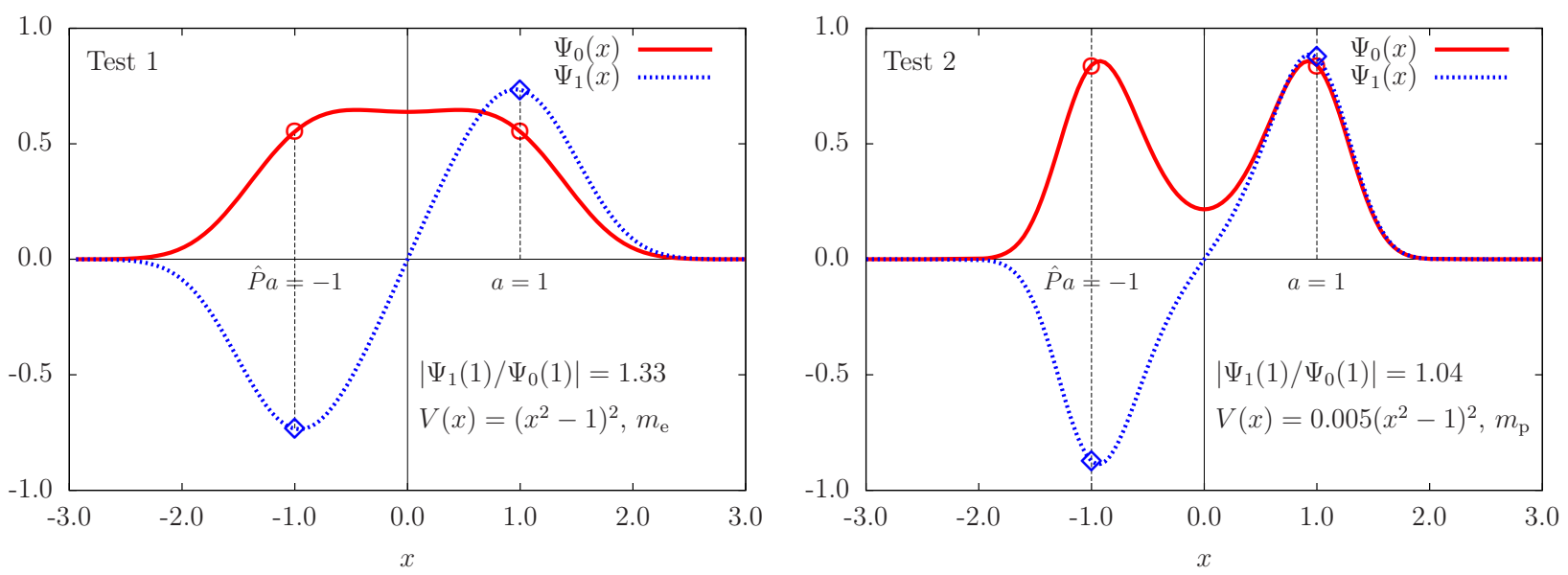

FIG. 2: Ground and first-excited state wave functions (calculated variationally) for the delocalized (Test 1) and localized (Test 2) one-dimensional double-well systems used to test the PIMD method. The points $a$ and $\hat{P} a$ indicate the positions of the fixed end-beads of the linear polymer in the PIMD simulations.

\section{NUMERICAL RESULTS}

\section{A. One-dimensional test calculations}

The linear-polymer path-integral molecular dynamics (PIMD) approach outlined in the previous sections was tested on one-dimensional model systems to allow comparison with the exact quantum result. We consider two one-dimensional test cases: Test 1 with $V(x)=\left(x^{2}-1\right)^{2}$ and $m=1$ and Test 2 with $V(x)=0.005\left(x^{2}-1\right)^{2}$ and $m=1822.888$ (we use atomic units unless otherwise stated). Test 1 corresponds to a system in which the barrier penetration is sufficient to delocalize the zero-point vibration above the barrier, and Test 2 to a system in which the penetration is less, giving a localized probability density (see Fig. 2).

A series of PIMD simulations were carried out with various simulation parameters (number of beads, time step, simulation time, coupling strength with the Andersen thermostat, etc.) to check the convergence properties of the method. Converged results for Test 1 (Table I) were obtained using $M=30-50$ beads, $N_{\xi}=5$ Gauss-Legendre integration points, simple Cartesian sampling (without mass scaling), a time step $\Delta t=0.001$, total simulation time $t_{\max }=10^{5}-10^{6}$, and a coupling frequency of 1000-10000 for the Andersen thermostat. More extensive tests were necessary for the Test 2 system, for which the greater degree of localization made convergence more difficult; we used the values of $M$ and $N_{\xi}$ given in Table II, used normal-mode sampling, with frequencies scaled to $\tilde{\Omega}=1$ [see Eq. (34)], $\Delta t=0.01$, 


\begin{tabular}{|c|c|c|c|c|c|c|c|}
\hline & $\rho(1$, & $1 ; \beta)$ & $/ \rho(1$ & $,-1 ; \beta)$ & $\Delta E$ & $\bar{\beta}(1)$ & $\Psi_{1}$ \\
\hline$\beta$ [a.u.]: & 3 & 4 & 5 & 10 & [a.u.] & [a.u.] & \\
\hline PIMD & 1.39 & 1.16 & 1.07 & 1.00 & 0.79 & 0.70 & 1.32 \\
\hline variational & 1.39 & 1.16 & 1.07 & 1.00 & 0.79 & 0.71 & 1.33 \\
\hline
\end{tabular}

TABLE I: Ground-state tunneling splittings $\Delta E$ and tunnelling times $\bar{\beta}(1)$ calculated for the one-dimensional 'Test 1' system by PIMD and variationally. Also shown are the wave function amplitude ratios obtained from $\bar{\beta}(1)$ using Eq. (8). For comparison, the tunneling-splitting estimate given by instanton theory $[17,22]$ is $\Delta E(\mathrm{RPI})=1.6$ a.u.

$t_{\max }=10^{6}$ a.u. and a coupling frequency of 1000 for the Andersen thermostat. In both sets of simulations, the fixed end-points of the linear polymer, $a=1$ and $\hat{P} a=-1$, were taken to be the minima of the two wells. The inverse of the $\rho(a, a ; \beta) / \rho(a, \hat{P} a ; \beta)$ values listed in Tables I and II were used to fit Eq. (6) to obtain $\Delta E$ and $\bar{\beta}(a)$ in each row (here $a=1$, and thus $\bar{\beta}(1)$ is obtained). Tables I and II also provide the wave function ratio calculated from Eq. (8), and compare with the variational results.

Tables I and II show that the PIMD splittings and ratios of the wave function amplitudes are in excellent agreement with the variational results. However, as would be expected, it is difficult to obtain tight convergence and high accuracy from the PIMD simulations. We analyzed the sensitivity of the fitted energy splitting with respect to errors in the density matrix ratios. The error in the datapoint corresponding to the largest $\beta$ value has by far the largest effect on the error of the fitted $\Delta E$ value; the lowest- $\beta$ point is also important (but less so). These two datapoints mainly fix the parameters of the hyperbolic tangent function Eq. (6); errors in the datapoints of the intermediate $\beta$ values have only a minor effect. In addition, it is also important to point out that for larger $\beta$ values it is more computationally demanding to converge the PIMD simulations (most importantly, larger $M$ values are necessary). Therefore, in practice, an optimal $\beta$ range has to be found: $\beta$ has to be large enough to sufficiently depopulate higher excited states in the sum, Eqs. (4)-(6), whilst remaining as low as possible to minimize the costs of the PIMD simulation.

In numerically integrating the potential of mean force, we have tested the Gauss-Legendre (GL) quadrature [56] and the Clenshaw-Curtis (CC) quadrature, first proposed in ref. [57]. The CC quadrature is a nested quadrature, which means that for special numbers of grid points the full CC grid includes smaller CC grids, and thereby it allows for the direct assessment of the error of the numerical integration from a single calculation, which is, 


\begin{tabular}{|c|c|c|c|c|c|c|c|c|c|c|}
\hline \multirow[b]{2}{*}{$\beta$ [a.u.]: } & \multicolumn{7}{|c|}{ 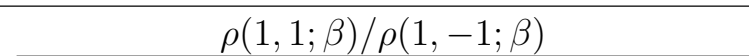 } & $\Delta E$ & $\bar{\beta}(1)$ & \multirow{2}{*}{$\mid \frac{\Psi_{1}(1)}{\Psi_{0}(1)}$} \\
\hline & 30003500 & 4000 & 4500 & 5000 & 10000 & 15000 & 20000 & {$\left[10^{-5}\right.$ a.u. } & [a.u.] & \\
\hline $\operatorname{PIMD}(50,5)$ & 12.09 .70 & 8.34 & 7.20 & 6.26 & 3.10 & 2.21 & 1.72 & 6.78 & 400 & 1.01 \\
\hline $\operatorname{PIMD}(50,10)$ & 11.59 .48 & 7.94 & 7.01 & 6.05 & 2.88 & 1.99 & 1.7 & 7.28 & 475 & 1.02 \\
\hline $\operatorname{PIMD}(100,10)$ & 11.79 .46 & 7.94 & 6.86 & 5.99 & 2.77 & 1.98 & 1.51 & 8.16 & 898 & 1.04 \\
\hline $\operatorname{PIMD}(150,10)$ & 11.79 .45 & 7.91 & 6.90 & 5.99 & 2.78 & 1.96 & 1.50 & 8.26 & 939 & 1.04 \\
\hline $\operatorname{PIMD}(200,10)$ & 11.79 .44 & 7.92 & 6.72 & 5.99 & 2.75 & 1.91 & 1.53 & 8.24 & 892 & 1.04 \\
\hline $\operatorname{PIMD}(200,15)$ & 11.79 .42 & 7.87 & 6.85 & 6.00 & 2.77 & 1.91 & 1.52 & 8.28 & 926 & 1.04 \\
\hline rariational & 11.79 .43 & 7.91 & 6.81 & 5.99 & 2.79 & 1.91 & 1.52 & 8.27 & 925 & 1.04 \\
\hline
\end{tabular}

TABLE II: Ground-state tunneling splittings $\Delta E$ and tunnelling times $\bar{\beta}(1)$ calculated for the one-dimensional 'Test 2' system by PIMD and variationally. Each row gives the $\Delta E$ obtained by fitting Eq. (6) to the density-matrix ratios obtained on the left $\left[\right.$ where ' $\operatorname{PIMD}\left(M, N_{\xi}\right)$ ' specifies the number of beads and Gauss-Legendre integration points]. Also shown are the wave function amplitude ratios obtained from $\bar{\beta}(1)$ using Eq. (8). For comparison, the tunneling-splitting estimate given by instanton theory $[17,22]$ is $\Delta E(\mathrm{RPI})=10^{-4}$ a.u.

in principle, an appealing feature. The GL quadrature provided quantitative or almost quantitative results with already $N_{\xi}=5$ points, and $N_{\xi}=10$ or $N_{\xi}=15$ points were sufficient in all test cases we considered. At the same time, it was always necessary to use more than $15-20$ points for the CC quadrature, and we had to use $N_{\xi}=31$ (or 61 grid points) to obtain the desired accuracy (these specific values have been selected because they include several smaller CC grids). Since, the overall cost of the computations scales linearly with the number of grid points (number of independent simulations), we continued using the GL quadrature with $N_{\xi}=5-10$ points (and validated our choice by carrying out a few computations with $N_{\xi}=15-20$ GL quadrature points).

\section{B. Malonaldehyde}

As a more realistic test, we applied the PIMD method to malonaldehyde, explicitly including all $f \times N=3 \times 9=27$ Cartesian coordinates in the simulation. Tunneling in malonaldehyde has a rich experimental [58-61] and theoretical [62-67] literature, but the focus here is on testing the PIMD method for this system. All calculations used the potential energy surface and fast analytic gradient routine recently published in ref. [68], which also gives the ground state tunneling-splitting for this potential energy surface (obtained from fixed-node DMC calculations).

In the PIMD calculations, the point $\boldsymbol{r}_{0}$ was taken to be the equilibrium geometry in the 

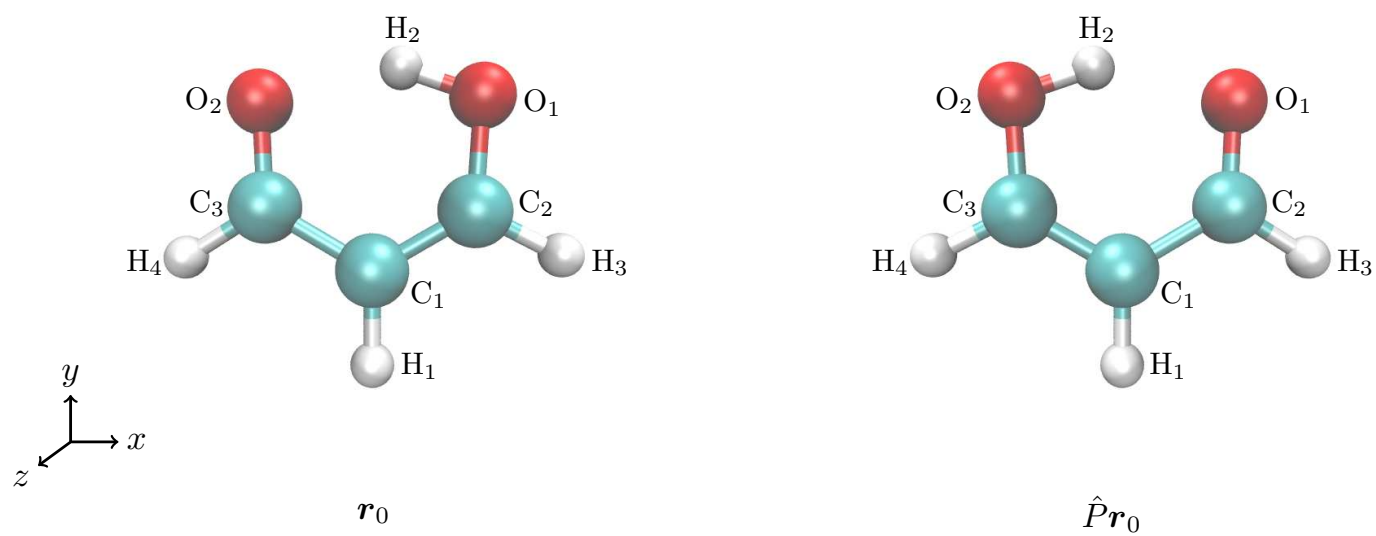

FIG. 3: The end-point geometries $\boldsymbol{r}_{0}$ and $\hat{P} \boldsymbol{r}_{0}$ used in the PIMD tunneling-splitting calculations on malonaldehyde (which correspond to the equilibrium geometries in the two wells in the principal axis frame); $\hat{P}=\hat{R}_{y, \pi}\left(\mathrm{O}_{1} \mathrm{O}_{2}\right)\left(\mathrm{C}_{2} \mathrm{C}_{3}\right)\left(\mathrm{H}_{3} \mathrm{H}_{4}\right)$. The Cartesian coordinates of $\boldsymbol{r}_{0}$ and $\hat{P} \boldsymbol{r}_{0}$ are provided in the Supplemental Material [69].

principal axis frame (see Fig. 3) with the center-of-mass at the origin, which ensured that the density matrix elements were free from any translational contribution. To simplify the calculation, we considered a single symmetry operation, $\hat{P}$, which was a pairwise exchange of equivalent atoms in the skeleton followed by a $180^{\circ}$ rotation around the $y$ axis. Thereby, the pair of structures $\boldsymbol{r}_{0}$ and $\hat{P} \boldsymbol{r}_{0}$ (see Fig. 3) corresponds to the 'proton jump' (and relaxation of the skeleton to the equilibrium structure). With this definition of the end points, we assume that the rotational contributions in the nominator and denominator of Eq. (6) factorize and cancel and we obtain a good approximation of the exact splitting for rotational quantum number $J=0$. The full treatment of the rotation-inversion-permutation symmetry is left for future work.

Numerical results are presented in Table III obtained using the following simulation parameters. In order to converge the propagator ratios within ca. $10 \%$, we used $M=$ $50-175$ beads $(M=50-100$ was sufficient for $\beta=3000$ but $M=150$ was necessary for $\beta=5000$ ). The potential of mean force was integrated numerically over a Gauss-Legendre quadrature with $N_{\xi}=10-20$. The dynamical equations were integrated in the scaled normal-mode representation (with $\tilde{\Omega}=1$ ) with a time step of $\Delta t=0.005-0.01$ a.u. for a total simulation time of $t_{\max }=1 \cdot 10^{5}-2 \cdot 10^{5}$ a.u. $(2-5 \mathrm{ps})$ coupled to an Andersen thermostat with a collision frequency of 1000. Data collection began after an initial equilibration time of $10 \%$ (or up to 50\%) of the total simulation time (longer for larger $\beta$ values and for end-points over the potential-energy-barrier corresponding to the middle windows of the 


\begin{tabular}{|c|c|c|c|c|c|c|c|c|}
\hline & $\rho\left(\boldsymbol{r}_{0}\right.$ & $\boldsymbol{r}_{0}$ & )$/ \rho($ & ${ }_{0}, \hat{P} \boldsymbol{r}$ & $0 ; \beta)$ & $\Delta E$ & $\bar{\beta}\left(\boldsymbol{r}_{0}\right)$ & \\
\hline$\beta$ [a.u.]: & 3000 & 3500 & 4000 & 4500 & 5000 & {$\left[\mathrm{~cm}^{-1}\right]$} & [a.u.] & $\Psi_{+}(\boldsymbol{r}$ \\
\hline PIMD, Fit-1 & 12.0 & 9.4 & 7.8 & & & 19.9 & $1.2 \cdot 10^{3}$ & 1.05 \\
\hline & 12.1 & 9.8 & 8.0 & & & 18.8 & $1.1 \cdot 10^{3}$ & 1.05 \\
\hline & & & & & & $19.4(5)$ & $1150(50)$ & $1.05(0)$ \\
\hline$\overline{\text { PIMD, Fit-2 }}$ & 12.0 & 9.4 & 7.8 & 6.4 & & 21.4 & $1.3 \cdot 10^{3}$ & 1.07 \\
\hline & 12.1 & 9.8 & 8.0 & 6.8 & & 19.2 & $1.1 \cdot 10^{3}$ & 1.05 \\
\hline & & & & & & $20.3(11)$ & $1200(100)$ & $1.06(1)$ \\
\hline$\overline{\text { PIMD, Fit-3 }}$ & 12.0 & 9.4 & 7.8 & 6.4 & 5.6 & 21.5 & $1.3 \cdot 10^{3}$ & 1.07 \\
\hline & 12.1 & 9.8 & 8.0 & 6.8 & 6.0 & 19.0 & $1.1 \cdot 10^{3}$ & 1.05 \\
\hline & & & & & & $20.3(13)$ & $1200(100)$ & $1.06(1)$ \\
\hline PIMD, Fit-4 & & 9.4 & 7.8 & 6.4 & & 22.3 & $1.4 \cdot 10^{3}$ & 1.07 \\
\hline & & 9.8 & 8.0 & 6.8 & & 20.1 & $1.3 \cdot 10^{3}$ & 1.06 \\
\hline & & & & & & $21.2(11)$ & $1350(50)$ & $1.07(1)$ \\
\hline$\overline{\text { PIMD, Fit-5 }}$ & & 9.4 & 7.8 & 6.4 & 5.6 & 21.9 & $1.4 \cdot 10^{3}$ & 1.07 \\
\hline & & 9.8 & 8.0 & 6.8 & 6.0 & 19.3 & $1.2 \cdot 10^{3}$ & 1.05 \\
\hline & & & & & & $20.6(13)$ & $1300(100)$ & $1.06(1)$ \\
\hline DMC [68] & & & & & & $21.0(4)$ & & \\
\hline
\end{tabular}

TABLE III: Tunnelling splittings $\Delta E$ of the rovibrational ground state of malonaldehyde calculated by PIMD using the PES of ref. [68]. Each value of $\Delta E$ and $\bar{\beta}\left(\boldsymbol{r}_{0}\right)$ was obtained by fitting Eq. (6) to the set of density-matrix ratios listed in the same row (labeled as Fit- $n$ ). The relative amplitude of the eigenfunctions is calculated from $\bar{\beta}\left(\boldsymbol{r}_{0}\right)$ using Eq. (8). An estimate of the sampling error is indicated by the upper and lower values for each dataset and is obtained by taking the deviation from the mean of the results obtained from the two datasets (see text for more details).

thermodynamic integration). We plan to improve the sampling efficiency (especially for the intermediate windows of the numerical integral) in later work, and therefore the error bounds reported in the Table correspond to the sampling uncertainty of these simulation windows (and are not derived from a rigorous statistical analysis). Within these uncertainties, the tunneling splittings obtained are in good agreement with the DMC result [68].

As to the computational efficiency of the PIMD simulations, approximately $10^{7} \mathrm{MD}$ integration steps for $1.5 \cdot 10^{2}$ beads in 10 thermodynamic integration windows (along a linearly interpolating reaction coordinate between the end structures) were carried out for at least two different $\beta$ values to obtain each value of $\Delta E$ in Table III. This corresponds to a total number of $6 \times 10^{10}$ potential energy gradient evaluations (there are two gradient evaluations in each MD integration step [55]). An energy gradient evaluation using the fast 
analytic gradient routines of ref. [68] took only approximately twice as long as a singlepoint energy evaluation, which made our simulations possible within a reasonable amount of computing time.

In comparison, DMC calculations [68] on the same potential energy surface used $5 \times 10^{3}$ walkers for $5.5 \times 10^{5}$ a.u. total (equilibration plus sampling) time with a (shortest) time step of 1 a.u. These calculations for both the ground and the excited state corresponded to a total number of $2 \times 2.75 \times 10^{9}=5.5 \times 10^{9}$ potential energy evaluations. Extrapolation to zero time step from a series of simulations and consideration of the fixed-node error allowed the authors to pinpoint a final error of $0.4 \mathrm{~cm}^{-1}$ for the ground-state tunneling splitting. Hence, the PIMD results of Table III were approximately 10 times as expensive as DMC, and gave an error that was roughly three times larger.

\section{SUMMARY AND OUTLOOK}

Test calculations on malonaldehyde including all 27 atomic degrees of freedom have demonstrated that PIMD is certainly a feasible method for calculating tunnelling splittings in molecules and clusters, but that, as currently implemented, it is less efficient than DMC, requiring ten times as many potential energy evaluations to converge the splittings to within three times the statistical error (as recent DMC calculations on the same surface [68]). Hence, in systems for which the nodal surface is known to a good approximation (e.g. malonaldehyde), it makes sense to use DMC to calculate the tunnelling splittings. However, in systems for which the nodal surface is difficult to determine (e.g. water clusters), the extra numerical effort required by PIMD may be a price worth paying.

In addition, it may be possible to improve the numerical efficiency of PIMD tunnelling splitting calculations. As currently implemented, the ratios of density matrix elements are computed by thermodynamic integration, along a pathway in which only one of the coordinates of one of the beads contributes to the statistical averages. Pathways with a more evenly distributed dependence on the beads might therefore lead to more efficient sampling, as might the use of umbrella integration [70], or suitably adapted versions of transition-path sampling [71] or metadynamics [72].

One can also expect that the PIMD method will become more efficient for facile tunnelling motions (e.g. the acceptor tunneling motion in water dimer), where less free energy 
is required to pull the linear polymer over the barrier, and where instanton methods such as RPI break down (since the fluctuations around the minimum-action paths are not then well described to second order). In this sense, the PIMD and RPI methods complement one another.

Additional further areas of development of the PIMD method could include extending it to treat tunnelling between multiple wells (which has been done already for instantons in the RPI method [23]), and relaxing the assumption that vibration-rotation coupling can be neglected. A generalization of this double-well approach to higher-order discrete and continuous symmetries is presented in Ref. [73].

\section{Acknowledgment}

EM would like to thank Michael Willatt, Conan Zhang, Adam Reid, and Jeremy Richardson for many insightful discussions. EM, DJW and SCA acknowledge funding from the UK Engineering and Physical Sciences Research Council. 


\section{Appendix A: Normal modes of the linear polymer}

The equations of motion corresponding to the free linear-polymer Hamiltonian, $H_{\boldsymbol{a}, \boldsymbol{b}}^{[M-1]}\left(\boldsymbol{p}, \boldsymbol{r} ; \beta_{M}\right)$ or for short $H^{[M-1]}$, Eq. (15), with equal bead masses, $\mu_{i}=\mu_{i}^{(1)}=\ldots=$ $\mu_{i}^{(M-1)}$, are

$$
\dot{r}_{i}^{(j)}=\frac{\partial H^{[M-1]}}{\partial p_{i}^{(j)}}=\frac{1}{\mu_{i}} p_{i}^{(j)} \quad \Rightarrow \mu_{i} \dot{r}_{i}^{(j)}=p_{i}^{(j)}
$$

and

$$
\dot{p}_{i}^{(j)}=-\frac{\partial H^{[M-1]}}{\partial r_{i}^{(j)}}=-\left[m_{i} \omega_{M}^{2}\left(r_{i}^{(j)}-r_{i}^{(j-1)}\right)-m_{i} \omega_{M}^{2}\left(r_{i}^{(j+1)}-r_{i}^{(j)}\right)\right]
$$

with $j=1,2, \ldots, M-1, i=1,2, \ldots, f N$ and with the fixed ends, $r_{i}^{(0)}=a_{i}$ and $r_{i}^{(M)}=b_{i}$.

$$
\begin{aligned}
\mu_{i} \ddot{r}_{i}^{(j)} & =-m_{i} \omega_{M}^{2}\left(2 r_{i}^{(j)}-r_{i}^{(j+1)}-r_{i}^{(j-1)}\right) \\
\ddot{r}_{i}^{(j)} & =-\frac{m_{i}}{\mu_{i}} \omega_{M}^{2}\left(2 r_{i}^{(j)}-r_{i}^{(j+1)}-r_{i}^{(j-1)}\right) \\
\ddot{r}_{i}^{(j)} & =-\tilde{\omega}_{M}^{2}\left(2 r_{i}^{(j)}-r_{i}^{(j+1)}-r_{i}^{(j-1)}\right) \quad \text { with } \quad\left[r_{i}^{(0)}=a_{i} \text { and } r_{i}^{(M)}=b_{i}\right]
\end{aligned}
$$

where $\tilde{\omega}_{M}=\tilde{\mu}^{1 / 2} \omega_{M}$ was introduced with $\tilde{\mu}=m_{i} / \mu_{i}$, which is chosen to be the same for all $i=1,2, \ldots, f N$.

By including the coordinates of the fixed end points explicitly, the equations of motion are

$$
\begin{aligned}
\ddot{r}_{i}^{(1)} & =-\tilde{\omega}_{M}^{2}\left(2 r_{i}^{(1)}-r_{i}^{(2)}-a_{i}\right) \quad(j=1) \\
\ddot{r}_{i}^{(j)} & =-\tilde{\omega}_{M}^{2}\left(2 r_{i}^{(j)}-r_{i}^{(j+1)}-r_{i}^{(j-1)}\right) \quad(j=2, \ldots, M-2) \\
\ddot{r}_{i}^{(M-1)} & =-\tilde{\omega}_{M}^{2}\left(2 r_{i}^{(M-1)}-b_{i}-r_{i}^{(M-2)}\right) \quad(j=M-1),
\end{aligned}
$$


which can be rearranged to

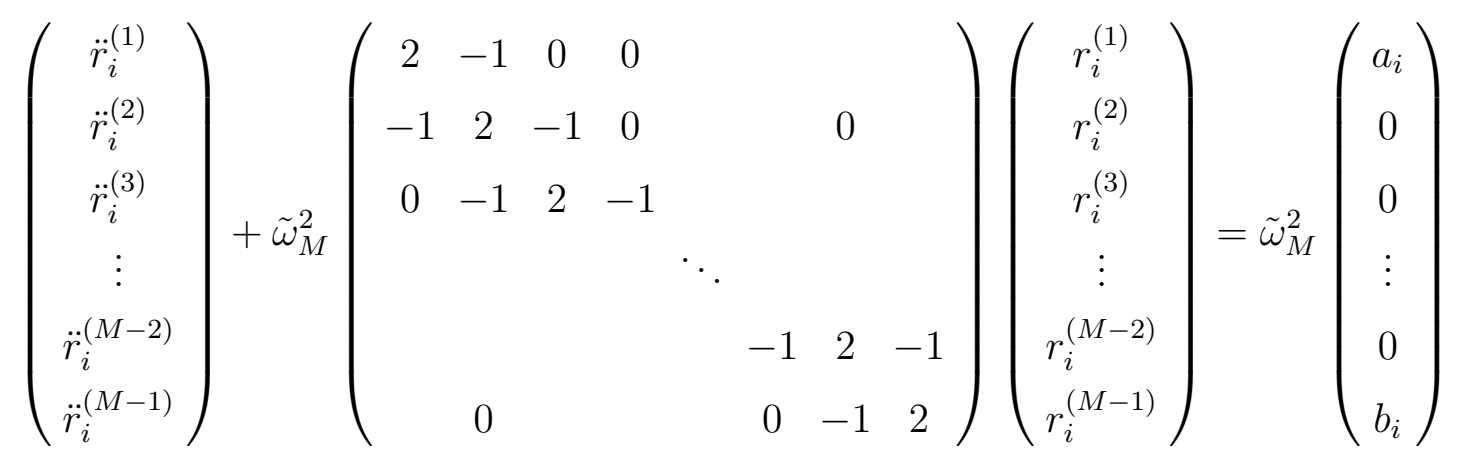

$$
\ddot{\boldsymbol{r}}_{i}^{(.)}+\tilde{\omega}_{M}^{2} \boldsymbol{A} \boldsymbol{r}_{i}^{(.)}=\tilde{\omega}_{M}^{2} \boldsymbol{c}_{i}^{(.)}
$$

where $\boldsymbol{r}_{i}^{(.)}$denotes a vector, which collects all (active) bead coordinates corresponding to $i$ th degree of freedom, $\boldsymbol{r}_{i}^{(.)^{\mathrm{T}}}=\left(r_{i}^{(1)}, r_{i}^{(2)}, \ldots, r_{i}^{(M-1)}\right)$ and $\boldsymbol{A} \in \mathbb{R}^{(M-1) \times(M-1)}$, and $\boldsymbol{c}_{i}^{(.)} \in \mathbb{R}^{M-1}$ are defined by Eqs. (A.7)-(A.8).

The eigenvalues and the normalized eigenvectors of $\boldsymbol{A}$ can be calculated analytically and they are given here without derivation (the solution is similar to that of the Hückel problem of linear polyenes). $\boldsymbol{O}^{\mathrm{T}} \boldsymbol{A} \boldsymbol{O}=\boldsymbol{\Lambda},(\boldsymbol{\Lambda})_{k j}=\delta_{k j} \lambda^{(j)}$ with

$$
O_{k j}=\sqrt{\frac{2}{M}} \sin \left(\frac{k j \pi}{M}\right) \quad j, k=1,2, \ldots, M-1
$$

and

$$
\lambda^{(j)}=4 \sin ^{2}\left(\frac{j \pi}{2 M}\right)
$$

Next, we multiply both sides of Eq. (A.8) with the orthogonal matrix $\boldsymbol{O}^{\mathrm{T}}$

$$
\begin{aligned}
\boldsymbol{O}^{\mathrm{T}} \ddot{\boldsymbol{r}}_{i}^{(.)}+\tilde{\omega}_{M}^{2} \boldsymbol{O}^{\mathrm{T}} \boldsymbol{A} \boldsymbol{r}_{i}^{(.)} & =\tilde{\omega}_{M}^{2} \boldsymbol{O}^{\mathrm{T}} \boldsymbol{c}_{i}^{(.)} \\
\boldsymbol{O}^{\mathrm{T}} \ddot{\boldsymbol{r}}_{i}^{(.)}+\tilde{\omega}_{M}^{2} \boldsymbol{O}^{\mathrm{T}} \boldsymbol{A} \boldsymbol{O} \boldsymbol{O}^{\mathrm{T}} \boldsymbol{r}_{i}^{(.)} & =\tilde{\omega}_{M}^{2} \boldsymbol{O}^{\mathrm{T}} \boldsymbol{c}_{i}^{(.)}
\end{aligned}
$$


and introduce $\boldsymbol{\rho}_{i}^{(.)}=\boldsymbol{O}^{\mathrm{T}} \boldsymbol{r}_{i}^{(.)}$and $\boldsymbol{\gamma}_{i}^{(.)}=\boldsymbol{O}^{\mathrm{T}} \boldsymbol{c}_{i}^{(.)}$

$$
\ddot{\boldsymbol{\rho}}_{i}^{(.)}+\tilde{\omega}_{M}^{2} \boldsymbol{\Lambda} \boldsymbol{\rho}_{i}^{(.)}=\tilde{\omega}_{M}^{2} \gamma_{i}^{(.)}
$$

and thus for $j=1,2, \ldots, M-1$ we write

$$
\begin{aligned}
\ddot{\rho}_{i}^{(j)}+\tilde{\omega}_{M}^{2} \lambda^{(j)} \rho_{i}^{(j)} & =\tilde{\omega}_{M}^{2} \gamma_{i}^{(j)} \\
\ddot{\rho}_{i}^{(j)} & =-\tilde{\omega}_{M}^{2} \lambda^{(j)}\left(\rho_{i}^{(j)}-\frac{1}{\lambda^{(j)}} \gamma_{i}^{(j)}\right)
\end{aligned}
$$

where we used that $\lambda^{(j)}$ is not zero. Next, we introduce $\eta_{i}^{(j)}=\rho_{i}^{(j)}-\gamma_{i}^{(j)} / \lambda^{(j)}$ and use that $\ddot{\eta}_{i}^{(j)}=\ddot{\rho}_{i}^{(j)}$ :

$$
\ddot{\eta}_{i}^{(j)}=-\tilde{\omega}_{M}^{2} \lambda^{(j)} \eta_{i}^{(j)} \quad j=1,2, \ldots, M-1
$$

Thus, for an $(M+1)$-bead linear polymer with the two end beads fixed at $\boldsymbol{r}^{(0)}=\boldsymbol{a}$ and $\boldsymbol{r}^{(M)}=\boldsymbol{b}$ the normal-mode angular frequencies are

$$
\Omega^{(j)}=2 \tilde{\omega}_{M} \sin \left(\frac{j \pi}{2 M}\right)
$$

with $\tilde{\omega}_{M}=\tilde{\mu}^{1 / 2} \omega_{M}$ and the corresponding normal coordinates are

$$
\begin{aligned}
\eta_{i}^{(j)}= & \rho_{i}^{(j)}-\gamma_{i}^{(j)} / \lambda^{(j)} \\
= & \left(\boldsymbol{O}^{\mathrm{T}} \boldsymbol{r}_{i}^{(.)}\right)_{j}-\left(\boldsymbol{O}^{\mathrm{T}} \boldsymbol{c}_{i}^{(.)}\right)_{j} / \lambda^{(j)} \\
= & \sum_{k=1}^{M-1} \sqrt{\frac{2}{M}} \sin \left(\frac{k j \pi}{M}\right) r_{i}^{(k)} \\
& \quad-\frac{1}{4 \sin ^{2}\left(\frac{j \pi}{2 M}\right)} \sqrt{\frac{2}{M}}\left[a_{i} \sin \left(\frac{j \pi}{M}\right)+b_{i} \sin \left(\frac{(M-1) j \pi}{M}\right)\right], \\
& \quad i=1,2, \ldots, f N \text { and } j=1,2, \ldots, M-1 .
\end{aligned}
$$

Since $\boldsymbol{O}$ is an orthogonal matrix, we can easily write down also the inverse of this transfor- 
mation

$$
\begin{aligned}
& r_{i}^{(k)}=\sum_{j=1}^{M-1} \sqrt{\frac{2}{M}} \sin \left(\frac{j k \pi}{M}\right) \\
& \times {\left[\eta_{i}^{(j)}+\frac{1}{4 \sin ^{2}\left(\frac{j \pi}{2 M}\right)} \sqrt{\frac{2}{M}}\left(a_{i} \sin \left(\frac{j \pi}{M}\right)+b_{i} \sin \left(\frac{(M-1) j \pi}{M}\right)\right)\right], } \\
& i=1,2, \ldots, f N \quad \text { and } \quad k=1,2, \ldots, M-1 .
\end{aligned}
$$

The summation in the first term of Eqs. (A.18) and (A.19), was implemented using a fast sine transform (related to a fast Fourier transform) algorithm. Finally, we also note that there is no zero-frequency mode for a linear polymer, Eq. (A.17) in contrast to the ring polymers (and analogously to the different energy-level pattern of the linear and cyclic polyenes).

[1] T. R. Walsh and D. J. Wales, J. Chem Soc., Faraday Trans. 92, 2505 (1996).

[2] T. Taketsugu and D. J. Wales, Mol. Phys. 100, 2793 (2002).

[3] Y. Watanabe, T. Taketsugu, and D. J. Wales, J. Chem. Phys. 120, 5993 (2004).

[4] M. Takahashi, Y. Watanabe, T. Taketsugu, and D. J. Wales, J. Chem. Phys. 123, 044302 (2005).

[5] M. A. Suhm and R. O. Watts, Phys. Rep. 204, 293 (1991).

[6] M. Quack and M. A. Suhm, J. Chem. Phys. 95, 28 (1991).

[7] M. Quack and M. A. Suhm, 234, 71 (1995).

[8] J. K. Gregory and D. C. Clary, J. Chem. Phys. 102, 7817 (1995).

[9] J. K. Gregory and D. C. Clary, J. Chem. Phys. 105, 6626 (1996).

[10] S. C. Althorpe and D. C. Clary, J. Chem. Phys. 101, 3603 (1994).

[11] S. C. Althorpe and D. C. Clary, J. Chem. Phys. 102, 4390 (1995).

[12] M. J. Smit, G. C. Groenenboom, P. E. S. Wormer, A. van der Avoird, R. Bukowski, and K. Szalewicz, J. Phys. Chem. A 105, 6212 (2001).

[13] X. Huang, B. J. Braams, J. M. Bowman, R. E. A. Kelly, J. Tennyson, G. C. Groenenboom, and A. van der Avoird, J. Chem. Phys. 128, 034312 (2008).

[14] C. Leforestier, K. Szalewicz, and A. van der Avoird, J. Chem. Phys. 137, 014305 (2012). 
[15] D. Blume and K. B. Whaley, J. Chem. Phys. 112, 2218 (2000).

[16] A. Viel, M. D. Coutinho-Neto, and U. Manthe, J. Chem. Phys. 126, 024308 (2007).

[17] A. I. Vainshtein, V. I. Zakharov, V. A. Novikov, and M. A. Shifman, Sov. Phys. Usp. 25, 195 (1982).

[18] G. V. Mil'nikov and H. Nakamura, J. Chem. Phys. 115, 6881 (2001).

[19] G. V. Mil'nikov, K. Yagi, T. Taketsugu, H. Nakamura, and K. Hirao, J. Chem. Phys. 119, 10 (2003).

[20] G. V. Mil'nikov, K. Yagi, T. Taketsugu, H. Nakamura, and K. Hirao, J. Chem. Phys. 120, 5036 (2004).

[21] G. V. Mil'nikov and H. Nakamura, J. Chem. Phys. 122, 124311 (2005).

[22] J. O. Richardson and S. C. Althorpe, J. Chem. Phys. 134, 054109 (2011).

[23] J. O. Richardson, S. C. Althorpe, and D. J. Wales, J. Chem. Phys. 135, 124109 (2011).

[24] J. O. Richardson, D. J. Wales, S. C. Althorpe, R. P. McLaughlin, M. R. Viant, O. Shih, and R. J. Saykally, J. Phys. Chem. A 117, 6960 (2013).

[25] E. Zwart, J. J. T. Meulen, W. L. Meerts, and L. H. Coudert, J. Mol. Spectrosc. 147, 27 (1991).

[26] F. N. Keutsch and R. J. Saykally, Proc. Nat. Acad. Sci. USA 98, 10533 (2001).

[27] F. N. Keutsch, J. D. Cruzan, and R. J. Saykally, Chem. Rev. 103, 2533 (2003).

[28] N. Pugliano and R. J. Saykally, Science 257, 1937 (1992).

[29] K. Liu, M. J. Elrod, J. G. Loeser, J. D. Cruzan, N. Pugliano, M. G. Brown, J. Rzepiela, and R. J. Saykally, Faraday Discuss. 97, 35 (1994).

[30] J. D. Cruzan, L. B. Braly, K. Liu, M. G. Brown, J. G. Loeser, and R. J. Saykally, Science 271, 59 (1996).

[31] K. Liu, M. G. Brown, J. D. Cruzan, and R. J. Saykally, Science 271, 62 (1996).

[32] K. Liu, M. G. Brown, C. Carter, R. J. Saykally, J. K. Gregory, and D. C. Clary, Nature 381, 501 (1996).

[33] C. Pérez, D. P. Zaleski, N. A. Seifert, B. Temelso, G. C. Shields, Z. Kisiel, and B. H. Pate, Angew. Chem. 126, 14596 (2014).

[34] J. B. Anderson, J. Chem. Phys. 63, 1499 (1975).

[35] V. Buch, J. Chem. Phys. 97, 726 (1992).

[36] A. B. McCoy, Int. Rev. Phys. Chem. 25, 77 (2006). 
[37] D. Blume, M. Lewerenz, P. Niyaz, and K. B. Whaley, Phys. Rev. E 55, 3664 (1997).

[38] D. Blume, M. Lewerenz, and K. B. Whaley, J. Chem. Phys. 107, 9067 (1997).

[39] M. T. Čvitas and S. C. Althorpe, J. Chem. Theory Comput. (2015).

[40] W. H. Miller, J. Chem. Phys. 62, 1899 (1975).

[41] J. B. Rommel and J. Kästner, J. Chem. Phys. 134, 184107 (2011).

[42] J. B. Rommel, T. P. M. Goumans, and J. Kästner, J. Chem. Theory Comput. 7, 690 (2011).

[43] Z. Smedarchina, W. Siebrand, and A. Fernández-Ramos, J. Chem. Phys. 137, 224105 (2012).

[44] D. Chandler and P. G. Wolynes, J. Chem. Phys. 74, 4078 (1981).

[45] E. L. Pollock and D. M. Ceperley, Phys. Rev. B 30, 2555 (1984).

[46] M. Parrinello and A. Rahman, J. Chem. Phys. 80, 860 (1984).

[47] C. Alexandrou and J. W. Negele, Phys. Rev. C 37, 1513 (1988).

[48] D. M. Ceperley and G. Jacucci, Phys. Rev. Lett. 58, 1648 (1987).

[49] D. M. Ceperley, Rev. Mod. Phys. 67, 279 (1995).

[50] A. Kuki and P. G. Wolynes, Science 236, 1647 (1987).

[51] M. Marchi and D. Chandler, J. Chem. Phys. 95, 889 (1991).

[52] R. W. Zwanzig, J. Chem. Phys. 22, 1420 (1954).

[53] D. J. Wales, J. Chem. Phys. 142, 130901 (2015).

[54] H. C. Andersen, J. Chem. Phys. 72, 2384 (1980).

[55] M. Ceriotti, M. Parrinello, T. E. Markland, and D. E. Manolopoulos, J. Chem. Phys. 133, $124104(2010)$.

[56] M. Abramowitz and I. A. Stegun, eds., Handbook of Mathematical Functions with Formulas, Graphs, and Mathematical Tables (Dover, New York, 1972).

[57] C. W. Clenshaw and A. R. Curtis, Numerische Mathematik 2, 197 (1960).

[58] S. L. Baughcum, Z. Smith, E. B. Wilson, and R. W. Duerst, J. Am. Chem. Soc. 106, 2260 (1984).

[59] T. Baba, T. Tanaka, I. Morino, K. M. T. Yamada, and K. Tanaka, J. Chem. Phys. 110, 4131 (1999).

[60] T. N. Wassermann, D. Luckhaus, S. Coussan, and M. A. Suhm, Phys. Chem. Chem. Phys. 8, $2344(2006)$.

[61] N. Lüttschwager, T. N. Wassermann, S. Coussan, and M. A. Suhm, Mol. Phys. 111, 2211 (2013). 
[62] M. D. Coutinho-Neto, A. Viel, and U. Manthe, J. Chem. Phys. 121, 9207 (2004).

[63] Y. Wang, B. J. Braams, J. M. Bowman, S. Carter, and D. P. Tew, J. Chem. Phys. 128, 224314 (2008).

[64] T. Hammer and U. Manthe, J. Chem. Phys. 134, 224305 (2011).

[65] M. Schröder, F. Gatti, and H.-D. Meyer, J. Chem. Phys. 134, 234307 (2011).

[66] T. Hammer and U. Manthe, J. Chem. Phys. 136, 054105 (2012).

[67] M. Schröder and H.-D. Meyer, J. Chem. Phys. 141, 034116 (2014).

[68] W. Mizukami, S. Habershon, and D. P. Tew, J. Chem. Phys. 141, 144310 (2014).

[69] See Supplementary Material Document No. for the detailed numerical results used for Figure 1 and Table 1.

[70] J. Kästner and W. Thiel, J. Chem. Phys. 123, 144104 (2005).

[71] P. G. Bolhuis, D. Chandler, C. Dellago, and P. L. Geissler, Ann. Rev. Phys. Chem. 53, 291 (2002).

[72] R. Quhe, M. Nava, P. Tiwary, and M. Parrinello, J. Chem. Theory Comput. 11, 1383 (2015).

[73] E. Mátyus and S. C. Althorpe, J. Chem. Phys. (2016). 